\title{
The Anti-Adhesive Effect of Curcumin on Candida albicans Biofilms on Denture Materials
}

\begin{abstract}
Hasanain Alalwan ${ }^{1,2}$, Ranjith Rajendran ${ }^{1}$, David F. Lappin ${ }^{1}$, Emilie Combet ${ }^{3}$, Muhammad Shahzad ${ }^{1,3,4}$, Douglas Robertson ${ }^{3}$, Christopher J. Nile ${ }^{1}$, Craig Williams ${ }^{5}$ and Gordon Ramage ${ }^{1 *}$
\end{abstract}

${ }^{1}$ Oral Sciences Research Group, Glasgow Dental School, University of Glasgow, Glasgow, UK, ${ }^{2}$ Department of Prosthodontics, College of Dentistry, University of Baghdad, Baghdad, Iraq, ${ }^{3}$ Department of Human Nutrition, School of Medicine, Dentistry and Nursing, College of Medical, Veterinary and Life Sciences, University of Glasgow, Glasgow, UK, ${ }^{4}$ Institute of Basic Medical Sciences, Khyber Medical University, Peshawar, Pakistan, ${ }^{5}$ Institute of Healthcare Policy and Practice, University of West of Scotland, Paisley, UK

The use of natural compounds as an alternative source of antimicrobials has become a necessity given the growing concern over global antimicrobial resistance. Polyphenols, found in various edible plants, offers one potential solution to this. We aimed to investigate the possibility of using curcumin within the context of oral health as a way of inhibiting and preventing the harmful development of Candida albicans biofilms. We undertook a series of adsorption experiments with varying concentrations of curcumin, showing that $50 \mu \mathrm{g} / \mathrm{ml}$ could prevent adhesion. This effect could be further synergized by the curcumin pre-treatment of yeast cells to obtain significantly greater inhibition (>90\%, $p<0.001$ ). Investigation of the biological impact of curcumin showed that it preferentially affected immature morphological forms (yeast and germlings), and actively promoted aggregation of the cells. Transcriptional analyses showed that key adhesins were down-regulated (ALS1 and ALS3), whereas aggregation related genes (ALS5 and AAF1) were up-regulated. Collectively, these data demonstrated that curcumin elicits anti-adhesive effects and that induces transcription of genes integrally involved in the processes related to biofilm formation. Curcumin and associated polyphenols therefore have the capacity to be developed for use in oral healthcare to augment existing preventative strategies for candidal biofilms on the denture surface.

\section{Keywords: Curcumin, polyphenol, Candida albicans, adhesion, adsorption}

\section{INTRODUCTION}

Increasingly there are fewer antimicrobial options available to treat life-threatening infections, due largely to therapeutic mismanagement actively driving resistance, coupled with disinvestment in antimicrobial drug development from the pharmaceutical industry. The antimicrobial resistance debate suggests an imminent return to an era of uncertainty and limited therapeutic options, suggesting that we should stop our wavering and tackle this threat head on.

Naturally derived chemotherapeutic agents are an attractive option, particularly those botanically derived molecules, which offer advantages over synthetic derivatives due to their natural evolution and diminished likelihood of resistance. An interesting active plant extract worth consideration is the polyphenol curcumin (diferuloylmethane), extracted from the 
rhizomes of the Curcuma longa plant (Mahmood et al., 2015). It is the active ingredient of turmeric and copiously used in Asia as a food additive or dietary supplement, though it forms typically $<5 \%$ composition (Esatbeyoglu et al., 2012; Kwon, 2014). Curcumin (CUR) shows safe and effective biological activities such as anti-inflammatory, antioxidant, anti-proliferation, with potential efficacy against many human diseases as suggested by animal studies (Gupta et al., 2012). Importantly, CUR displays broad-spectrum antimicrobial properties (Moghadamtousi et al., 2014), including antibacterial (Shahzad et al., 2015; Tyagi et al., 2015) and antifungal properties (Martins et al., 2009; Khan et al., 2012), as well as the ability to influence adhesive and biofilm properties (Shahzad et al., 2014, 2015).

Studies by our group have shown that CUR has the capacity to alter the adhesion of key periodontal pathogens, and impact overall biofilm formation (Shahzad et al., 2015). Parallel studies on the primary denture pathogen Candida albicans demonstrated that CUR exhibited anti-biofilm properties at high concentrations, as well as antifungal activity against planktonic and biofilm cells (Shahzad et al., 2014). It is thought that these elevated concentrations directly impact cell wall permeability through signaling of the MAP kinase and calcineurin-mediated signaling, pathways which maintains cell wall integrity (Kumar et al., 2014). C. albicans is a major global opportunistic pathogen, armed with recognized virulence determinants that include colonization factors (adhesins, hyphae and thigmotropic properties), as well as the release of invasins, such as hydrolytic proteins that facilitate invasion into the host (O'Donnell et al., 2015c). The ability to adhere to both biological and inert substrates and form biofilms makes this organism of particular interest in the context of oral disease (O'Donnell et al., 2016). Biofilm etiology in this environment is a primary mechanism of persistence and survival in the oral cavity, providing physical protection from endogenous and exogenous antimicrobial factors (Ramage et al., 2014). Most significantly, C. albicans prominent role in inducing inflammation to cause denture induced stomatitis means we have a keen interest in developing ways to manipulate and interfere with biofilm development, as this is critical in preventing this disease. Therefore, this study aimed to investigate whether CUR could be used through direct interaction with materials and the yeast C. albicans to interfere with early adhesion events on a clinically relevant substrate.

\section{MATERIALS AND METHODS}

\section{Culture Conditions and Standardization}

The laboratory based C. albicans SC5314 was used in this study (O'Donnell et al., 2016). Yeast cells were cultivated as working stocks on fresh Sabouraud agar (Sigma-Aldrich, UK) for $48 \mathrm{~h}$ at $30^{\circ} \mathrm{C}$ and maintained at $4^{\circ} \mathrm{C}$. One unique colony was used to grow the cells in yeast-peptone-dextrose (YPD) medium (SigmaAldrich) for $18 \mathrm{~h}$ at $30^{\circ} \mathrm{C}$ and $150 \mathrm{rpm}$ orbital shaker. The cells were washed twice by centrifuging in sterile phosphate buffered saline (PBS; Sigma-Aldrich, UK) and standardized using a Neubauer haemocytometer.

\section{Antifungal Susceptibility Testing}

Planktonic and sessile cells were first investigated for their susceptibility to the polyphenol CUR (HPLC grade, Acros Organics, Belgium). Stock CUR was prepared immediately preceding the experiment using an non-antimicrobial concentration of dimethyl sulfoxide (DMSO) as a solvent and adjusted to $<5 \% \mathrm{v} / \mathrm{v}$ in RPMI-1640 medium (SigmaAldrich, UK; Shahzad et al., 2015). Standardized CLSI M-27A broth microdilution methodology was initially undertaken for planktonic yeast cultures in 96 well round bottomed microtitre plates (CLSI-M27-A, 2008). Clear wells with no visible growth were considered as the minimum inhibitory concentration (MIC). For sessile susceptibility testing, pre-formed $24 \mathrm{~h}$ biofilms were challenged with CUR using standardized sessile antifungal testing (Ramage et al., 2001; Pierce et al., 2008). Reduction of tetrazolium to formazan through an XTT assay was used, and the optical densities quantified at $492 \mathrm{~nm}$ using a microtitre plate reader (FluoStar Omega, BMG Labtech, UK). Negative and positive controls were included, and the experimental wells data were compared to the positive control data to reveal the $\mathrm{SMIC}_{80}$, where the optical density is reduced more than $80 \%$ in comparison to the positive control optical density, reflecting significant bioactivity against the biofilm. These procedures were repeated in three independent occasions where three replicates have been considered.

\section{Investigating the Effect of CUR Adsorption on Adhesion}

The potential capability of CUR to be adsorbed to denture material was investigated. Heat cure poly methyl methacrylate (PMMA) denture base material (Chaperlin and Jacobs Ltd, Surrey, UK) was used to fabricate $12 \mathrm{~mm}$ diameter discs using the dental compression molding technique. These discs were immersed in $\mathrm{ddH}_{2} \mathrm{O}$ for 7 days to ensure excess toxic monomers were removed. CUR was diluted in RPMI-1640 medium to 200, 400, and $800 \mu \mathrm{g} / \mathrm{ml}$. Discs were distributed in 24 wells plates (Costar, Corning Incorporated, USA) and $1 \mathrm{ml}$ of the CUR suspension was added. The plates were incubated at room temperature for a series of time points $(1,5,10,30$, $60,120,240$, and $1,440 \mathrm{~min})$. Next, discs were transferred to fresh wells and washed with $1 \mathrm{ml}$ of distilled water and then $1 \mathrm{ml}$ of DMSO was added to dissolve the adsorbed CUR. To quantify the released CUR, a standard curve (0.39-100 $\mu \mathrm{g} / \mathrm{ml}$ serially double diluted) was developed and measured at $436 \mathrm{~nm}$ using the spectrophotometer. Based on these data, PMMA discs were immersed in $1 \mathrm{ml}$ of $800 \mu \mathrm{g} / \mathrm{ml}$ of CUR for $10 \mathrm{~min}$ (equivalent to $50 \mu \mathrm{g} / \mathrm{ml}$ ) then washed with PBS to remove the unabsorbed molecules. These treated discs were inoculated with $1 \mathrm{ml}$ of $5 \times 10^{5} \mathrm{CFU}$ of C. albicans SC5314 cells and incubated for $30 \mathrm{~min}$ at $37^{\circ} \mathrm{C}$. Following the initial adhesion, cells were washed in PBS and the adherent cells removed by sonication at $35 \mathrm{kHz}$ for $10 \mathrm{~min}$ (Ultrasonic bath, Fisher scientific, UK), and enumerated using the Miles and Misra plate counting method (Miles et al., 1938). The final cell number was expressed per $\mathrm{cm}^{2}$ PMMA, which was compared to a CUR negative control. All experiments were performed 
with three independent sections on three independent occasions. Scanning electron microscopy (SEM) was also performed using the same experimental parameters, then processed and imaged, as described previously by our group. Briefly, biofilms were grown on Thermanox ${ }^{\mathrm{TM}}$ coverslips or hydrogel cellulose matrix and treated, as previously described. Biofilms were washed twice with PBS, before being fixed in 2\% para-formaldehyde, $2 \%$ glutaraldehyde, $0.15 \mathrm{M}$ sodium cacodylate, and $0.15 \% \mathrm{w} / \mathrm{v}$ alcian blue, at pH 7.4, and prepared for SEM as previously described (Erlandsen et al., 2004). The specimens were sputter-coated with gold and viewed under a JEOL JSM-6400 scanning electron microscope.

\section{Investigating the Biological Effect of CUR on Adhesion and Biofilm Formation}

Discs were adsorbed with CUR as described above, then the discs (untreated and treated) were inoculated with either $1 \mathrm{ml}$ of $5 \times$ $10^{5} \mathrm{CFU}+3 \mathrm{~min}$ CUR $(50 \mu \mathrm{g} / \mathrm{ml})$ or PBS (negative control) treated C. albicans SC5314 cells, and incubated for $30 \mathrm{~min}$ at $37^{\circ} \mathrm{C}$. Adhesion of $C$. albicans was then assessed and quantified as described in the previous section, with the levels of adhesion expressed as a proportion of the negative control (PMMA-/C. albicans-). In parallel we then assessed whether longer CUR exposure time negatively impacted adhesion by treating cells for 3, 30, and $90 \mathrm{~min}$, and the levels of adhesion to PMMA quantified. Finally, we assessed whether or not CUR exposure to C. albicans cells at different growth phases played a role, with the hypothesis that there may be differences in how yeast $(Y)$, germlings $(G)$, or hyphae $(\mathrm{H})$ responded to this molecule. Briefly, cells were grown overnight, standardized to $1 \times 10^{6} \mathrm{CFU}$ in RPMI and inoculated into a 96-well microtitre plate. Cells were then exposed to 50, 100, or $200 \mu \mathrm{g} / \mathrm{ml}$ CUR at either $0 \mathrm{~h}(\mathrm{Y}), 2 \mathrm{~h}(\mathrm{G})$, or $4 \mathrm{~h}(\mathrm{H})$ postinoculation and incubated for a further $24 \mathrm{~h}$ at $37^{\circ} \mathrm{C}$. Thereafter, the cells were washed in PBS and the resultant biofilm quantified using an XTT metabolic reduction assay. All experiments were performed in duplicate on three independent occasions.

\section{Investigating the Aggregative Effect of CUR}

In order to assess whether CUR has additional effects on the physicality of these yeasts, we assessed its impact on aggregation. C. albicans SC5314 cells $(\mathrm{Y})$ were standardized $\left(1 \times 10^{6}\right.$ cells $)$ in PBS and exposed \pm to subinhibitory concentrations of CUR $(50 \mu \mathrm{g} / \mathrm{ml})$ for $90 \mathrm{~min}$ at $37^{\circ} \mathrm{C}$ under constant agitation $(200$ $\mathrm{rpm})$. Following incubation the cells were serially 10 diluted in PBS diluent and plated onto Sabouraud agar using the Miles and Misra methodology. The plates were then incubated overnight at $37^{\circ} \mathrm{C}$ and the colonies enumerated. In parallel, cells were examined under a light microscope to evaluate aggregation visually. All experiments were performed in triplicate on three independent occasions.

\section{Investigating the Molecular Effect of CUR on Adhesion and Biofilm Formation}

Preparation of $\mathrm{Y}$ and $\mathrm{H}$ cells was performed using an initial inoculum of $1 \times 10^{8}$ cells and $5 \times 10^{5}$ cells of C. albicans
SC5314 in RPMI, respectively. For H cells these were incubated on PMMA sections within 24 wells plates for $4 \mathrm{~h}$. Both Y and $\mathrm{H}$ cells were then treated \pm CUR $(50 \mu \mathrm{g} / \mathrm{ml})$ in RPMI for 3, 30, and $90 \mathrm{~min}$, after which they were prepared for RNA extraction. Cells were either centrifuged or sonicated in a $35 \mathrm{kHz}$ for $10 \mathrm{~min}$ (Ultrasonic bath, Fisher scientific, UK) to harvest the cells. These were then washed by centrifugation prior to RNA extraction using a combined mechanical disruption (0.5 mm glass beads) and chemical TRIzol ${ }^{\mathrm{TM}}$ method (Invitrogen, Paisley, UK). After DNase treatment (Qiagen, Crawley, UK) and purification (RNeasy MinElute clean up kit, Qiagen, Crawley, UK), cDNA was synthesized using a High Capacity RNA to cDNA kit (Life Technologies, Paisley, UK), and quantitative PCR performed using a SYBR ${ }^{\circledR}$ GreenER ${ }^{\mathrm{TM}}$ assay (Life Technologies Ltd, Paisley, UK). The primers used for quantitative PCR were ALS1, ALS3, ALS5 (agglutanin-like sequence 1, 3, and 5), EAP1 (epithelial adhesion protein 1), and AAF1 (adhesion and aggregation factor 1). Each parameter was analyzed in duplicate using MxProP Quantitative PCR machine and MxProP 3000 software (Stratagene, Amsterdam, Netherlands). Gene expression was normalized to the housekeeping gene ACT1 according to $2^{-\Delta \Delta C T}$ method (Livak and Schmittgen, 2001). Table 1 summarizes all the primer details used in this study. A heatmap was created for the differential expression of genes $\left(\log _{2}\right)$ over the period of 3-90 min from the untreated control compared to CUR exposed cells. Maps and clusters were generated in $\mathrm{R}$ with the use of heatmap 0.2 function from the gplots package. All experimnets were performed in triplicate on three independent occasions.

\section{Statistical Analysis}

As we were unable to ascertain that the data conformed to a Gaussian distribution data analysis was performed on non-parametric data using either a Mann-Whitney test or a Kruskal-Wallis test with Dunn's multiple comparison posttest. All independant data points are presented, with error bars representing the median with interquartile range. Where

\begin{tabular}{|c|c|c|}
\hline Gene & Sequence $\left(5^{\prime}-3^{\prime}\right)$ & References \\
\hline \multirow[t]{2}{*}{ ALS1 } & F-TTCTCATGAATCAGCATCCACAA & Nailis et al., 2009 \\
\hline & R-CAGAATTTCACCCATACTTGGTTC & \\
\hline \multirow[t]{2}{*}{ ALS3 } & F-CAACTTGGGTTATTGAAACAAAAACA & Nailis et al., 2009 \\
\hline & R-AGAAACAGAAACCCAAGAACAACCT & \\
\hline \multirow[t]{2}{*}{ ALS5 } & F-CTGCCGGTTATCGTCCATITA & $\begin{array}{l}\text { Green et al., } \\
2005\end{array}$ \\
\hline & R-ATTGATACTGGTTATTATCTGAGGGAGAAA & \\
\hline \multirow[t]{2}{*}{ EAP1 } & F-ACCACCACCGGGTATACAAA & $\begin{array}{l}\text { Sherry et al., } \\
2014\end{array}$ \\
\hline & R-GCCATCACATTTGGTGACAG & \\
\hline \multirow[t]{2}{*}{ AAF1 } & F-CTGCCCTTGTTGGTACATCT & This study \\
\hline & R-TGGGATAGTTGGTGGAGGAG & \\
\hline \multirow[t]{2}{*}{ ACT1 } & F-AAGAATTGATTTGGCTGGTAGAGA & $\begin{array}{l}\text { Ricardo et al., } \\
2009\end{array}$ \\
\hline & R-TGGCAGAAGATTGAGAAGAAGTT & \\
\hline
\end{tabular}


proportional data is presented, analysis was performed on the original data sets. All statistics and figures were produced using GraphPad Prism v.5 (GraphPad Software Inc., La Jolla, CA).

\section{RESULTS}

\section{Curcumin Adsorption Reduces Candida albicans Adhesion}

First, we tested the potential of CUR to inhibit and kill planktonic and biofilm cells to establish biologically active working concentrations suitable for use in downstream analyses. The planktonic MIC (PMIC) was shown to be $100 \mu \mathrm{g} / \mathrm{ml}$ of CUR for SC5314 and two other clinical strains tested (data not shown), whereas the sessile (biofilm) MIC that caused an $80 \%$ reduced metabolic activity $\left(\mathrm{SMIC}_{80}\right)$ was $\geq 200 \mu \mathrm{g} / \mathrm{ml}$, demonstrating that the biofilm's activity and/or viabilty are significantly reduced.

Based on these data we wanted to evaluate whether these levels of CUR could be adsorbed to PMMA material to prevent C. albicans adhesion. We therefore adsorbed 200, 400, and 800 $\mu \mathrm{g} / \mathrm{ml}$ of CUR to PMMA sections over different time periods and quantified these adsorbed concentrations using an elution method alongside an optimized standard curve. Figure 1A illustrates the kinetics of adsorption for each concentration. It was shown that $800 \mu \mathrm{g} / \mathrm{ml}$ CUR was required to achieve concentrations with anti-biofilm activity $(200 \mu \mathrm{g} / \mathrm{ml})$, though 90 min adsorption was required to achieve this. Nevertheless, after 10 and $30 \mathrm{~min}$ adsorption, 50 and $100 \mu \mathrm{g} / \mathrm{ml}$ concentrations were achieved from this initial concentration, respectively. The lower concentration of $400 \mu \mathrm{g} / \mathrm{ml}$ was able to achieve PMIC levels, though this took $\sim 90 \mathrm{~min}$ adsorption. Finally, $200 \mu \mathrm{g} / \mathrm{ml}$ was not able to achieve any antimicrobial level concentrations, even after $24 \mathrm{~h}$ adsorption. Based on these data we focussed on adsorption of $800 \mu \mathrm{g} / \mathrm{ml}$ for $10 \mathrm{~min}$, which was able to achieve $50 \mu \mathrm{g} / \mathrm{ml}$ on the surface of the PMMA for downstream analysis. Next, we evaluated the capacity of $C$. albicans to attach to PMMA for 30 min adsorbed with CUR $(50 \mu \mathrm{g} / \mathrm{ml})$ and compared a control (Figure 1B). Here we showed a significant three-fold reduction in adhesion of $C$. albicans was observed $(p<0.004)$. When analyzed by SEM the visible reduction of yeasts cells can be shown on the PMMA surfaces (Figure 1C).

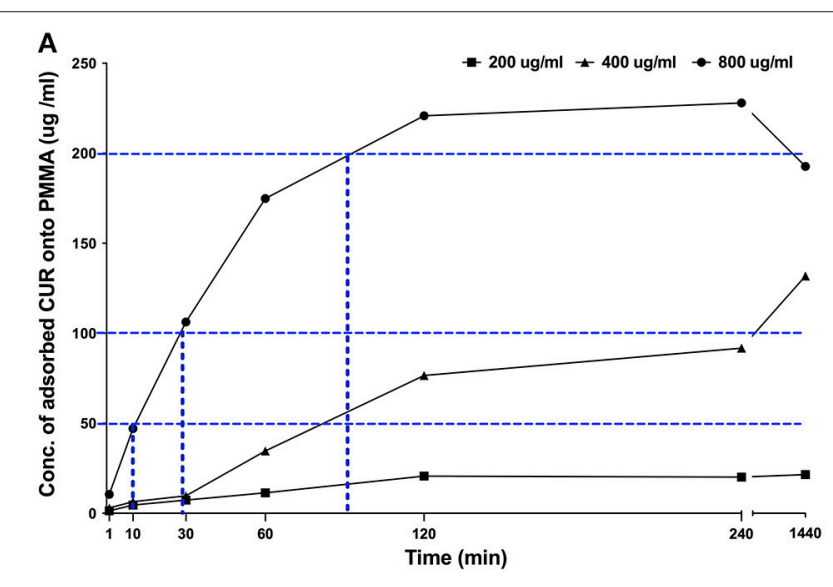

C

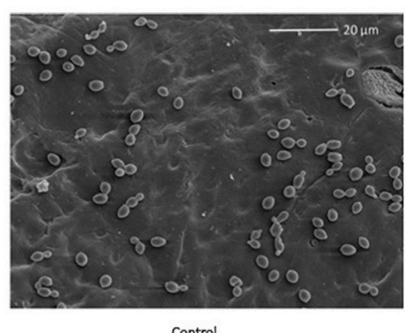

Control

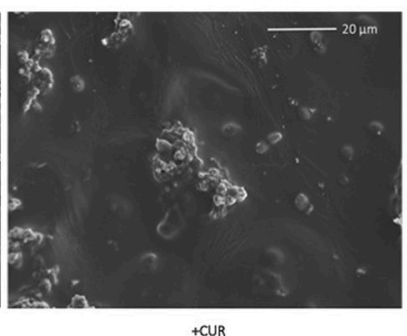

+CUR

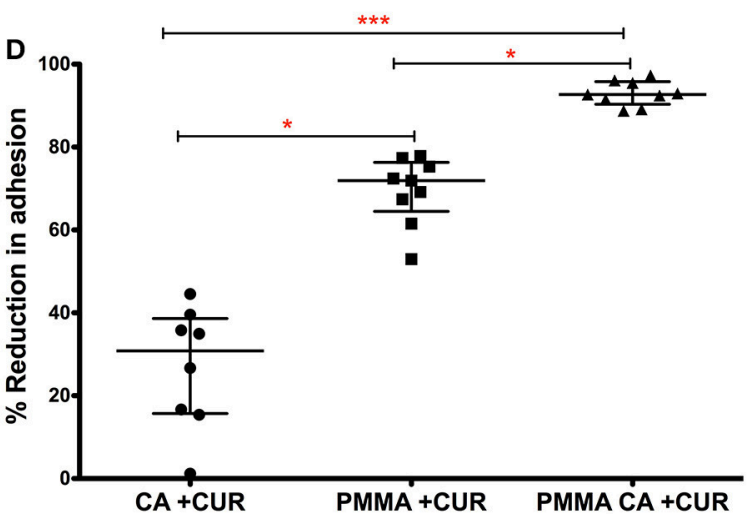

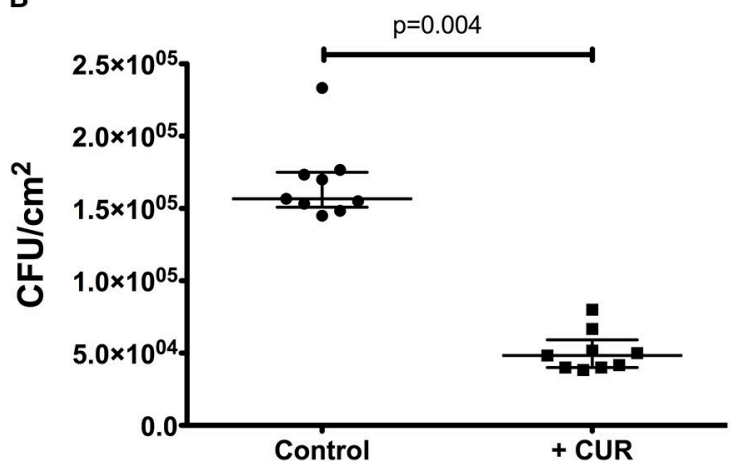

FIGURE 1 | The impact of CUR adsorption to PMMA and its impact on Candida albicans SC5314 adhesion. (A) Time and concentration dependant adsorption of CUR to PMMA (blue dotted line) at half the MIC (50 $\mu \mathrm{g} / \mathrm{ml})$, MIC (100 $\mu \mathrm{g} / \mathrm{ml})$, and SMIC $80200 \mu \mathrm{g} / \mathrm{ml}$. (B) C. albicans inhibitory capability of $50 \mu \mathrm{g} / \mathrm{ml}$ adsorbed CUR onto PMMA compared to untreated control and a Mann-Whitney test was performed on data from nine independent experiments. (C) SEM images of 30 min adherent C. albicans cells onto CUR adsorbed (+CUR) and non-adsorbed PMMA control. (D) Single and dual-treatment of CUR on C. albicans adhesion, which were analyzed using a Kruskal-Wallis test with Dunn's multiple comparison post-test performed on data from nine independent experiments. All independant data points are presented, with error bars representing the median with interquartile range $\left({ }^{\star} p<0.05,{ }^{* \star} p<0.01,{ }^{* \star *} p<0.001\right)$. 
Given that CUR was shown to elicit antimicrobial effects, both planktonically and to a lesser extent against sessile biofilms cells, and that adsorption appeared to influence adhesive capacity, we decided to investigate whether combining an early direct effect on C. albicans with that of adsorption to PMMA would synergize CUR activity in prevention of adhesion and colonization. $C$. albicans was treated for $3 \mathrm{~min} \pm$ CUR $50 \mu \mathrm{g} / \mathrm{ml}$ (CA + CUR), which was compared to both PMMA adsorbed with CUR (PMMA + CUR), or a combination of CUR treated C. albicans and CUR adsorbed PMMA (PMMA CA + CUR). Figure 1D demonstrates that the direct treatment of $C$. albicans alone only inhibited $30 \mathrm{~min}$ adhesion by $27 \%$, which was significantly lower than CUR adsorption alone $(70 \%, p<0.05)$. However, combining the effect on $C$. albicans with adsorbed CUR resulted in a significant reduction than adsorption alone of $93 \%(p<0.001)$, thus improving the anti-adhesive capacity of C. albicans.

\section{Curcumin Prevents Biofilm Formation and Promotes Candida albicans Aggregation}

Our data above showed a positive anti-candidal effect with respect to surface adsorption, but also showed that a brief CUR pre-exposure ( $3 \mathrm{~min}$ ) resulted in reduces adhesion of $C$. albicans, suggesting sub-inhibitory concentrations elicited some biological activity. We therefore sought to further investigate this effect on $C$. albicans by extending the CUR pre-exposure time. We were able to show that extending the time from 3 to 30 and 90 min significantly enhanced anti-adhesion $(p>0.01)$. Though, between 30 and $90 \mathrm{~min}$ there were no significant improvements in anti-adhesion properties (Figure 2A).

In order to understand the longer term effects of CUR treatment on $C$. albicans and how this could impact biofilm formation, we prepared yeast cells $(\mathrm{Y}=0 \mathrm{~min})$, germlings $(\mathrm{G}=$ $120 \mathrm{~min})$ and hyphae $(\mathrm{H}=240 \mathrm{~min})$ prior to CUR exposure at 50,100 , and $200 \mu \mathrm{g} / \mathrm{ml}$, which were then allowed to develop for biofilm for $24 \mathrm{~h}$ (Figure 2B). The resultant data showed that at lower sub-inhibitory concentrations $(50 \mu \mathrm{g} / \mathrm{ml})$ and PMIC levels $(100 \mu \mathrm{g} / \mathrm{ml})$ the anti-biofilm effects moderately impacted the overall biofilm metabolism, though at SMIC levels $(200 \mu \mathrm{g} / \mathrm{ml})$ a significant reduction in biofilm formation was observed for $\mathrm{Y}$ and $\mathrm{G}$ to approximately $>90 \%$ of the control $(p<0.01)$, though $\mathrm{H}$ cells were least impacted $(p<0.05)$.

Next, we sought to determine whether CUR exhibited any additional effects on C. albicans. The premise of the experiment was to assess whether CUR induced aggregation through
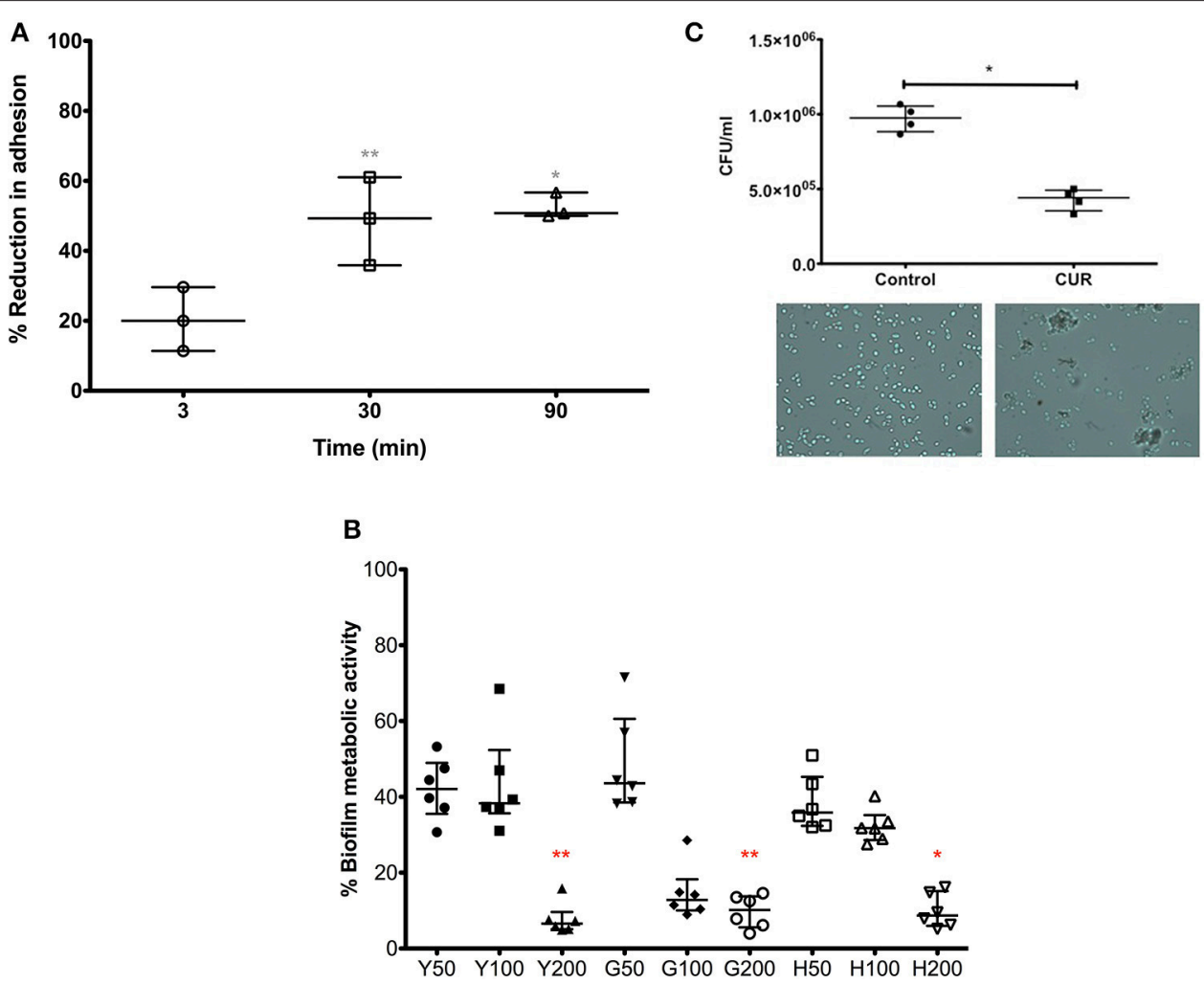

FIGURE 2 | The impact of CUR on C. albicans adhesion, biofilm formation, and aggregation. (A) C. albicans was pre-treatment with sub-inhibitory concentration of CUR (50 $\mu \mathrm{g} / \mathrm{ml})$ for 3, 30, and $90 \mathrm{~min}$ and adhesion to PMMA assessed on data from triplicate data from three independent experiments. (B) Different morphological forms of $C$. albicans (Y, yeast; $\mathrm{G}$, germlings; $\mathrm{H}$, hyphae) were pre-treated with $\mathrm{CUR}$ at 50,100 , and $200 \mu \mathrm{g} / \mathrm{ml}$, and the resultant biofilm formation assessed metabolically after $24 \mathrm{~h}$. Data represents six independent experiments, which was analyzed using a Kruskal-Wallis test with Dunn's multiple comparison post-test. (C) Aggregation of C. albicans exposed to CUR (50 $\mu \mathrm{g} / \mathrm{ml})$ was assessed by total viable cell counts, analyzed using a Mann-Whitney test on triplicate data from four independent experiments, and the phenotype validated by light microscopy (400 $\times$ magnification). All independent data points are presented, with error bars representing the median with interquartile range $\left({ }^{*} p<0.05,{ }^{* *} p<0.01,{ }^{* *} p<0.001\right)$. 
alteration of the cell wall surface. We reasoned that using a plate counting based approach of cells treated at sub-inhibitory concentrations, then if aggregation occurred then the CFU would be lower than the respective control as each aggregate would result in only one CFU due to a heterogeneous population of cells. Indeed, we demonstrated a significant reduction in cell counts in the CUR group $(p<0.01)$, which was further confirmed through light microscopy observations (Figure 2C).

\section{Curcumin Affects the Temporal Expression of Candida albicans Adhesins}

Our data above suggest that CUR elicits biological effects on C. albicans, most significantly on preventing biofilm formation and impacting aggregation. We therefore aimed to assess a panel of associated genes through transcriptional analysis. To do this we focussed on $\mathrm{Y}$ and $\mathrm{H}$ cells at 3, 30, and 90 min post CUR exposure. Visual representation of these patterns was illustrated using heat map analysis and hierarchical clustering (Figure 3; raw $\mathrm{C}_{T}$ data profiles are presented in Supplementary Figure 1). We demonstrated that Y cells treated with CUR showed temporal changes in gene expression, most notably the down-regulation of the adhesin ALS3, and minimal impact on its related ALS1. Whereas, the clustered aggregative and flocculation genes AAF1, EAP1, and ALS5 transcripts were all up-regulated in a time dependant manner. Similar patterns and clustering of expression were also observed for the $\mathrm{H}$ cells, with AAF1 showing the highest levels of expression at $30 \mathrm{~min}$ compared to the control, and reciprocally ALS3 being the most down-regulated. Overall though the levels of differential expression were consistently lower in the H cells (Figure 3B) than Y cells (Figure 3A).

\section{DISCUSSION}

CUR is a polyphenol with potent biological effects, and has been described as a modern biological regulator (Esatbeyoglu et al.,
2012). The data presented herein demonstrate the potential for its use within the context of oral health for denture wearers. We have shown that CUR has the capacity to adsorb to denture relevant substrates and to inhibit $C$. albicans adhesion, rather than actively kill or inhibit the microorganisms. Interestingly, $C$. albicans exposed to CUR induced cellular aggregation, and effect that also reduced its adhesion capacity. Transcriptional analyses revealed that key adhesins were negatively impacted whereas genes associated with aggregation were positively impacted. Collectively, these data demonstrate that CUR has the potential to be used in denture care as a means of preventing dentureinduced stomatitis, a disease associated with C. albicans biofilms (O’Donnell et al., 2015a).

Initially, we wanted to evaluate and confirm the antimicrobial properties of CUR. Our data is in general agreement with others, showing that concentrations around $100 \mu \mathrm{g} / \mathrm{ml}$ are required to inhibit cellular growth (Martins et al., 2009; Sharma et al., 2010; Khan et al., 2012), and $200 \mu \mathrm{g} / \mathrm{ml}$ to elicit any antibiofilm activity (Shahzad et al., 2014). Any deviations can be accounted for in terms of variability of CUR source and purity, ratios of curcuminiods involved, and protocols used for broth microdilution method and the strains used. Given that our primary interest was in preventing C. albicans opposed to actively inhibiting and killing $C$. albicans then we focussed on lower sub-inhibitory concentrations of $50 \mu \mathrm{g} / \mathrm{ml}$. This was driven through the translational possibility that CUR could be taken as part of a diet or supplement, so could be maintained within saliva as well as adsorbing to hard tissues and prostheses in the oral cavity. Here, we showed that $\sim 6 \%$ of the CUR provided adsorped to the surface within $10 \mathrm{~min}$. Conceptually, CUR could be delivered directly during ingestion, and then back through the blood into saliva indirectly. In Nepal and India for example, daily CUR consumption can reach up to $100 \mathrm{mg}$ in Nepal and India (Shahzad et al., 2015), whereas in South Korea this may only reach $15 \mathrm{mg}$ (Kwon, 2014).
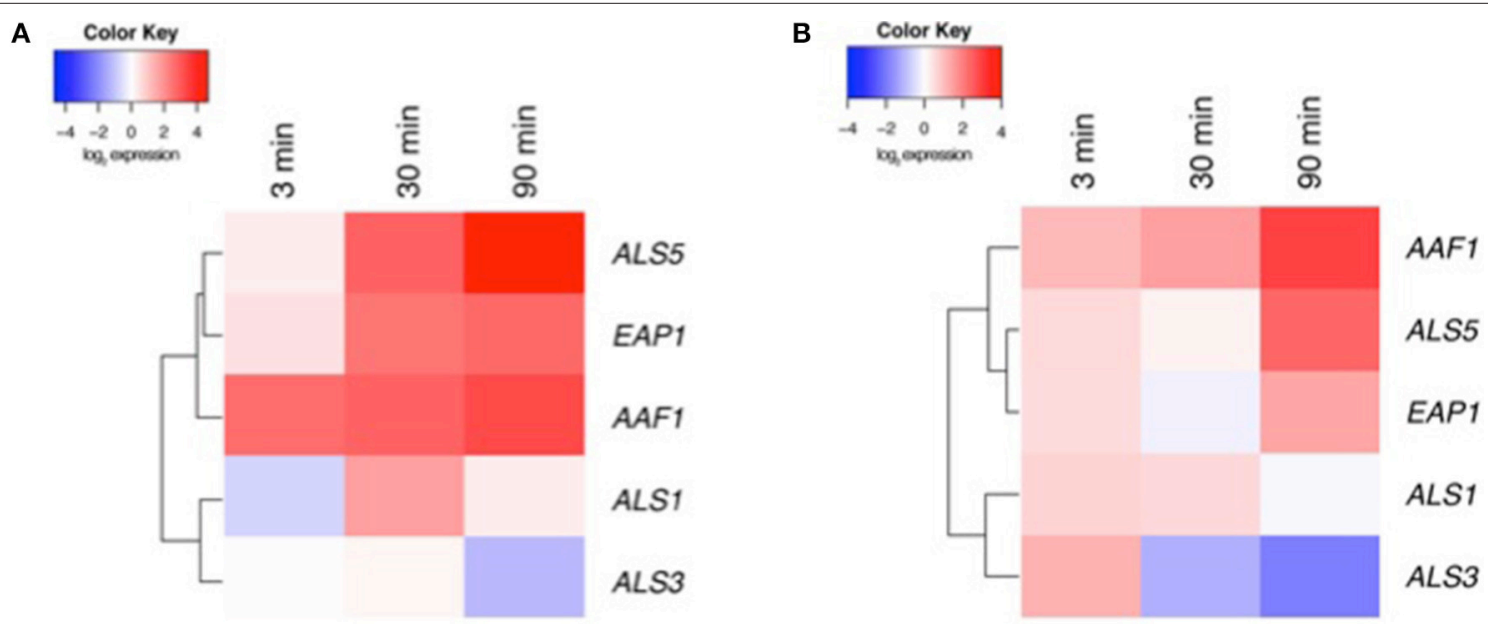

FIGURE 3 | Transcriptional analysis of CUR treated C. albicans. (A) Y and (B) H cells were prepared and exposed \pm CUR for 3,30 , and 90 min. Expression of ALS1, ALS3, ALS5, EAP1, and AAF1 were then assessed using qPCR and relative gene expression assessed the ACT1 housekeeping gene. A heatmap and clustering was created for the differential expression of genes $\left(\log _{2}\right)$. 
So delivering an antimicrobial concentration solely through a diet is not without challenges, though reaching an antiadhesive concentration is possible. To this end we were able to demonstrate that $50 \mu \mathrm{g} / \mathrm{ml}$ could be readily adsorbed onto PMMA, the polymer used to construct denture prostheses. The optimized PMMA adsorbed with CUR was shown to reduce $C$. albicans adhesion by up to $70 \%$, which was further reduced by $93 \%$ when a $3 \mathrm{~min}$ pre-treated C. albicans were inoculated on the optimized CUR adsorbed PMMA denture material. This synergized effect demonstrated that CUR has dual functionality, through surface adsorption and directly against $C$. albicans.

To further understand the biological basis of the subinhibitory CUR effect, we undertook a series of experiments to determine its effects on the kinetics of adhesion, whilst also evaluating how its impact on different morphological forms of C. albicans. We have previously shown that other natural compounds, such as tea tree oil (TTO) derivatives and carbohydrate-derived fulvic acid (CHD-FA) affect C. albicans development depending on the stage of biofilm growth (Ramage et al., 2012; Sherry et al., 2012), therefore we reasoned that CUR could also interact with $C$. albicans in a similar manner. We were able to show that prolonged exposure $(30 \mathrm{~min})$ of $C$. albicans yeast cells significantly reduced its adhesive capacity onto PMMA, suggesting that CUR was able to modify its adhesive capacity in some way. We further investigated this through looking at cells grown to different stages of morphological maturation, namely $\mathrm{Y}, \mathrm{G}$, and $\mathrm{H}$ cells. We hypothesized that depending on the stage of growth that the cells were exposed to CUR then this may affect overall biofilm development. Indeed, we showed that all morphological forms displayed reduced overall biofilm formation, though only the $200 \mu \mathrm{g} / \mathrm{ml}$ significantly reduced the $\mathrm{Y}$ and $\mathrm{G}$ cells in comparison to the other concentrations. $\mathrm{H}$ cells were not affected in a concentration dependant manner, suggesting that CUR was more effective against immature morphological forms. Interestingly, although biofilm formation was generally inhibited compared to control levels, there was still significant biofilm remaining, again suggesting early preventative intervention was most beneficial. The major limitation of this interpretation is the sample sizes used during these analyses (Vaux, 2012). Indeed, it begs the question whether the statistical analyses are worthwhile, hence why individual data-points are presented. Nevertheless, when we look at the data in its entirety, there are certainly trends suggestive that CUR exhibits positive biological effects, though further studies are required to confirm our observations.

CUR is a polyphenol with both antioxidant and hydrophobic properties (Priyadarsini, 2013; Mirzaei et al., 2017), which may explain why it preferentially adsorbs to PMMA and the cell wall of $C$. albicans. We hypothesized that the hydrophobic nature of the molecule could drive the coated $C$. albicans cells to aggregate with one another, and if we consider this within the context oral delivery within saliva then there is the possibility of creating complexes of cells that minimize their interaction with the denture surface. Indeed, we were able to demonstrate this both quantitatively and visually, which may explain why we observe synergized inhibition of adhesion at sub-inhibitory concentrations. C. albicans possesses a range of morphological and genetic attributes suited to colonization and biofilm formation (Blankenship and Mitchell, 2006). Finding ways of impacting these offers possibilities for novel anti-candidal therapeutics. Mechanistically, we were intrigued to understand how CUR induced C. albicans specific effects. Previous studies have shown that CUR has the ability to modulate of global repressor of filamentation TUP1 (Sharma et al., 2010). Indeed, our own focussed studies on mature biofilms showed that HWP1, a key hyphal wall associated element, was down-regulated (Shahzad et al., 2014). To this end we employed a transcriptional approach to assess genes implicated in adhesion and aggregation. CUR appeared to down-regulate the ALS agglutanins (ALS1 and ALS3), in both $\mathrm{Y}$ and $\mathrm{H}$ cells, suggesting it minimized their adhesive capacity. Both genes have been shown to be important in early biofilm events (Nailis et al., 2009; Fox et al., 2015). Of these ALS3 appeared to the most affected in these cells, which encodes the protein with a superior adhesive role and pivotal role in biofilm formation (Zhao et al., 2004; Nobile and Mitchell, 2005; Hoyer et al., 2008). ALS5 is a less well-defined member of this family, and although defined as an adhesin, functionally it appears to have amyloid properties and the capacity to improve aggregation (Rauceo et al., 2004; Garcia et al., 2011). This may explain why it is up-regulated following CUR treatment and this fits with the phenotype we observe. Moreover, AAF1 was also up-regulated by CUR in both $\mathrm{Y}$ and $\mathrm{H}$ cells, which is a gene highly related to the aggregation and flocculation (Fu et al., 1998). Interestingly it appears to have a minimal role in adhesion (Rieg et al., 1999), so further supports the notion of the phenotypes and anti-adhesive properties observed following induction by CUR. EAP1 showed similar trends to ALS5, though this encodes a protein known to enhance adhesion and biofilm formation (Li et al., 2007; Fox et al., 2015). This data is somewhat surprising, as we would have expected a similar level of down-regulation to that of ALS3. This suggests that EAP1, while exhibiting these adhesive properties, may have supplementary roles in cell-cell adhesion, though this requires further investigation. Collectively, these data demonstrate that CUR has the capacity at low concentrations to induce meaningful biological effects beneficial to minimizing candidal colonization.

In summary, given the strict denture hygiene regimen of brushing and cleansing required by an aging denture wearing global population, then this research provides opportunities to augment existing oral health strategies through dietary intake of important polyphenols like CUR. Not only is CUR effective against C. albicans, but also other oral pathogens (Shahzad et al., 2015). Given that the microbiome and mycobiome of denture wearers is highly diverse (O'Donnell et al., 2015b), then the broad-spectrum profile increases the overall appeal of this oral healthcare strategy. Indeed, there is merit to consider using a CUR based solution as a denture soak that has the potential to act and minimize further adhesion of these pathogenic biofilm related microorganisms, which with the enhanced antimicrobial activity induced through photactivation (Cieplik et al., 2015), may provide both a dual decontamination and preventative strategy. Indeed, clinical studies have already shown an additional benefit to photodynamic therapy (PDT) alone (Pereira et al., 
2015), as well as alongside CUR in the context of oral health (Leite et al., 2014). Mechanistically PTD works through locally acting light-activated photoantimicrobials molecules that produce highly reactive oxygen species, which are harmful to the site of action (Wainwright et al., 2017). This approach could therefore provide an augmentative benefit in enhancing lower concentrations of orally delivered antimicrobials, which could be light-activated bi-daily or more frequently. The hurdles will be in establishing and maintaining activatable concentrations that continue to exert an anti-adhesive effect. Careful consideration on the delivery of these molecules is required, and partnering up with nanotechnological approaches seems an obvious avenue of investigation, such as the creation of nanosized curcumin, which has already been shown to improve cellular interaction (Gopal et al., 2016), and would optimize our ability to deliver biologically relevant concentrations.

\section{AUTHOR CONTRIBUTIONS}

HA, RR, and MS participated in study design and experimental procedures and were responsible for preparation of the

\section{REFERENCES}

Blankenship, J. R., and Mitchell, A. P. (2006). How to build a biofilm: a fungal perspective. Curr. Opin. Microbiol. 9, 588-594. doi: 10.1016/j.mib.2006. 10.003

Cieplik, F., Pummer, A., Regensburger, J., Hiller, K. A., Spath, A., Tabenski, L., et al. (2015). The impact of absorbed photons on antimicrobial photodynamic efficacy. Front. Microbiol. 6:706. doi: 10.3389/fmicb.2015.00706

CLSI-M27-A (2008). CLSI Reference Method for Broth Dilution Antifungal Susceptibility Testing of Yeast; Approved Standard-3rd Edn. Clinical Laboratory Standards Institute. CLSI document M27-A3.

Erlandsen, S. L., Kristich, C. J., Dunny, G. M., and Wells, C. L. (2004). Highresolution visualization of the microbial glycocalyx with low-voltage scanning electron microscopy: dependence on cationic dyes. J. Histochem. Cytochem. 52, 1427-1435. doi: 10.1369/jhc.4A6428.2004

Esatbeyoglu, T., Huebbe, P., Ernst, I. M., Chin, D., Wagner, A. E., and Rimbach, G. (2012). Curcumin-from molecule to biological function. Angew. Chem. Int. Ed Engl. 51, 5308-5332. doi: 10.1002/anie.201107724

Fox, E. P., Bui, C. K., Nett, J. E., Hartooni, N., Mui, M. C., Andes, D. R., et al. (2015). An expanded regulatory network temporally controls Candida albicans biofilm formation. Mol. Microbiol. 96, 1226-1239. doi: 10.1111/mmi.13002

Fu, Y., Filler, S. G., Spellberg, B. J., Fonzi, W., Ibrahim, A. S., Kanbe, T., et al. (1998). Cloning and characterization of CAD1/AAF1, a gene from Candida albicans that induces adherence to endothelial cells after expression in Saccharomyces cerevisiae. Infect. Immun. 66, 2078-2084.

Garcia, M. C., Lee, J. T., Ramsook, C. B., Alsteens, D., Dufrene, Y. F., and Lipke, P. N. (2011). A role for amyloid in cell aggregation and biofilm formation. PLoS ONE 6:e17632. doi: 10.1371/journal.pone.0017632

Gopal, J., Muthu, M., and Chun, S. C. (2016). Water soluble nanocurcumin extracted from turmeric challenging the microflora from human oral cavity. Food Chem. 211, 903-909. doi: 10.1016/j.foodchem.2016.05.140

Green, C. B., Zhao, X., and Hoyer, L. L. (2005). Use of green fluorescent protein and reverse transcription-PCR to monitor Candida albicans agglutinin-like sequence gene expression in a murine model of disseminated candidiasis. Infect. Immun. 73, 1852-1855. doi: 10.1128/IAI.73.3.1852-1855.2005

Gupta, S. C., Patchva, S., Koh, W., and Aggarwal, B. B. (2012). Discovery of curcumin, a component of golden spice, and its miraculous biological activities. Clin. Exp. Pharmacol. Physiol. 39, 283-299. doi: $10.1111 /$ j.1440-1681.2011.05648.x manuscript. DL consulted and performed the statistical analysis $\mathrm{CW}, \mathrm{DR}$ and $\mathrm{CN}$ contributed to study design and manuscript preparation. GR and EC conceived the study, participated in study design and was responsible for producing the final manuscript. All authors have read and approved the final manuscript.

\section{ACKNOWLEDGMENTS}

HA is supported by the Iraqi Ministry of Higher Education and Scientific Research. RR is supported by the Wellcome Trust Strategic Award for Medical Mycology and Fungal Immunology 097377/Z/11/Z. We thank Margaret Mullin from the University of Glasgow for her expert assistance with scanning electron microscopy.

\section{SUPPLEMENTARY MATERIAL}

The Supplementary Material for this article can be found online at: http://journal.frontiersin.org/article/10.3389/fmicb. 2017.00659/full\#supplementary-material
Hoyer, L. L., Green, C. B., Oh, S. H., and Zhao, X. (2008). Discovering the secrets of the Candida albicans agglutinin-like sequence (ALS) gene family-a sticky pursuit. Med. Mycol. 46, 1-15. doi: 10.1080/13693780701435317

Khan, N., Shreaz, S., Bhatia, R., Ahmad, S. I., Muralidhar, S., Manzoor, N., et al. (2012). Anticandidal activity of curcumin and methyl cinnamaldehyde. Fitoterapia 83, 434-440. doi: 10.1016/j.fitote.2011.12.003

Kumar, A., Dhamgaye, S., Maurya, I. K., Singh, A., Sharma, M., and Prasad, R. (2014). Curcumin targets cell wall integrity via calcineurin-mediated signaling in Candida albicans. Antimicrob. Agents Chemother. 58, 167-175. doi: 10.1128/AAC.01385-13

Kwon, Y. (2014). Estimation of curcumin intake in Korea based on the Korea National Health and Nutrition Examination Survey (2008-2012). Nutr. Res. Pract. 8, 589-594. doi: 10.4162/nrp.2014.8.5.589

Leite, D. P., Paolillo, F. R., Parmesano, T. N., Fontana, C. R., and Bagnato, V. S. (2014). Effects of photodynamic therapy with blue light and curcumin as mouth rinse for oral disinfection: a randomized controlled trial. Photomed. Laser Surg. 32, 627-632. doi: 10.1089/pho.2014.3805

Li, F., Svarovsky, M. J., Karlsson, A. J., Wagner, J. P., Marchillo, K., Oshel, P., et al. (2007). Eaplp, an adhesin that mediates Candida albicans biofilm formation in vitro and in vivo. Eukaryot. Cell 6, 931-939. doi: 10.1128/EC.00049-07

Livak, K. J., and Schmittgen, T. D. (2001). Analysis of relative gene expression data using real-time quantitative PCR and the $2^{-\Delta \Delta C}$ T Method. Methods 25, 402-408. doi: 10.1006/meth.2001.1262

Mahmood, K., Zia, K. M., Zuber, M., Salman, M., and Anjum, M. N. (2015). Recent developments in curcumin and curcumin based polymeric materials for biomedical applications: a review. Int. J. Biol. Macromol. 81, 877-890. doi: 10.1016/j.ijbiomac.2015.09.026

Martins, C. V., Da Silva, D. L., Neres, A. T., Magalhaes, T. F., Watanabe, G. A., Modolo, L. V., et al. (2009). Curcumin as a promising antifungal of clinical interest. J. Antimicrob. Chemother. 63, 337-339. doi: 10.1093/jac/dkn488

Miles, A. A., Misra, S. S., and Irwin, J. O. (1938). The estimation of the bactericidal power of the blood. J. Hyg. 38, 732-749. doi: 10.1017/S002217240001158X

Mirzaei, H., Shakeri, A., Rashidi, B., Jalili, A., Banikazemi, Z., and Sahebkar, A. (2017). Phytosomal curcumin: a review of pharmacokinetic, experimental and clinical studies. Biomed. Pharmacother. 85, 102-112. doi: 10.1016/j.biopha.2016.11.098

Moghadamtousi, S. Z., Kadir, H. A., Hassandarvish, P., Tajik, H., Abubakar, S., and Zandi, K. (2014). A review on antibacterial, antiviral, and antifungal activity of curcumin. Biomed Res. Int. 2014:86864. doi: 10.1155/2014/186864. 
Nailis, H., Vandenbroucke, R., Tilleman, K., Deforce, D., Nelis, H., and Coenye, T. (2009). Monitoring ALS1 and ALS3 gene expression during in vitro Candida albicans biofilm formation under continuous flow conditions. Mycopathologia 167, 9-17. doi: 10.1007/s11046-008-9148-6

Nobile, C. J., and Mitchell, A. P. (2005). Regulation of cell-surface genes and biofilm formation by the C. albicans transcription factor Bcr1p. Curr. Biol. 15, 1150-1155. doi: 10.1016/j.cub.2005.05.047

O’Donnell, L. E., Alalwan, H. K., Kean, R., Calvert, G., Nile, C. J., Lappin, D. F., et al. (2016). Candida albicans biofilm heterogeneity does not influence denture stomatitis but strongly influences denture cleansing capacity. J. Med. Microbiol. 66, 54-60. doi: 10.1099/jmm.0.000419

O’Donnell, L. E., Millhouse, E., Sherry, L., Kean, R., Malcolm, J., Nile, C. J., et al. (2015a). Polymicrobial Candida biofilms: friends and foe in the oral cavity. FEMS Yeast Res. 15:fov077. doi: 10.1093/femsyr/fov077

O’Donnell, L. E., Robertson, D., Nile, C. J., Cross, L. J., Riggio, M., Sherriff, A., et al. (2015b). The oral microbiome of denture wearers is influenced by levels of natural dentition. PLoS ONE 10:e0137717. doi: 10.1371/journal.pone.0137717

O’Donnell, L. E., Robertson, D., and Ramage, G. (2015c). "Candida virulence factors," in Oral Candidosis: Physiopathology, Decision Making, and Therapeutics, ed E. A. R. Rosa (Berlin; Heidelberg: Springer-Verlag), 7-19.

Pereira, C. A., Domingues, N., Silva, M. P., Costa, A. C., Junqueira, J. C., and Jorge, A. O. (2015). Photodynamic inactivation of virulence factors of Candida strains isolated from patients with denture stomatitis. J. Photochem. Photobiol. B Biol. 153, 82-89. doi: 10.1016/j.jphotobiol.2015.08.029

Pierce, C. G., Uppuluri, P., Tristan, A. R., Wormley, F. L. Jr., Mowat, E., Ramage, G., et al. (2008). A simple and reproducible 96-well plate-based method for the formation of fungal biofilms and its application to antifungal susceptibility testing. Nat. Protoc. 3, 1494-1500. doi: 10.1038/nprot.2008.141

Priyadarsini, K. I. (2013). Chemical and structural features influencing the biological activity of curcumin. Curr. Pharm. Des. 19, 2093-2100. doi: 10.2174/1381612811319110010

Ramage, G., Milligan, S., Lappin, D. F., Sherry, L., Sweeney, P., Williams, C., et al. (2012). Antifungal, cytotoxic, and immunomodulatory properties of tea tree oil and its derivative components: potential role in management of oral candidosis in cancer patients. Front. Microbiol. 3:220. doi: 10.3389/fmicb.2012.00220

Ramage, G., Robertson, S. N., and Williams, C. (2014). Strength in numbers: antifungal strategies against fungal biofilms. Int. J. Antimicrob. Agents 43, 114-120. doi: 10.1016/j.ijantimicag.2013.10.023

Ramage, G., Vande Walle, K., Wickes, B. L., and Lopez-Ribot, J. L. (2001). Standardized method for in vitro antifungal susceptibility testing of Candida albicans biofilms. Antimicrob. Agents Chemother. 45, 2475-2479. doi: 10.1128/AAC.45.9.2475-2479.2001

Rauceo, J. M., Gaur, N. K., Lee, K. G., Edwards, J. E., Klotz, S. A., and Lipke, P. N. (2004). Global cell surface conformational shift mediated by a Candida albicans adhesin. Infect. Immun. 72, 4948-4955. doi: 10.1128/IAI.72.9.4948-49 55.2004

Ricardo, E., Costa-De-Oliveira, S., Dias, A. S., Guerra, J., Rodrigues, A. G., and Pina-Vaz, C. (2009). Ibuprofen revertsantifungal resistance on Candida albicans showing overexpression of CDR genes. FEMS Yeast Res. 9, 618-625. doi: 10.1111/j.1567-1364.2009.00504.X

Rieg, G., Fu, Y., Ibrahim, A. S., Zhou, X., Filler, S. G., and Edwards, J. E. (1999). Unanticipated heterogeneity in growth rate and virulence among Candida albicans AAF1 null mutants. Infect. Immun. 67, 3193-3198.

Shahzad, M., Millhouse, E., Culshaw, S., Edwards, C. A., Ramage, G., and Combet, E. (2015). Selected dietary (poly)phenols inhibit periodontal pathogen growth and biofilm formation. Food Funct. 6, 719-729. doi: 10.1039/C4FO01087F

Shahzad, M., Sherry, L., Rajendran, R., Edwards, C. A., Combet, E., and Ramage, G. (2014). Utilising polyphenols for the clinical management of Candida albicans biofilms. Int. J. Antimicrob. Agents 44, 269-273. doi: 10.1016/j.ijantimicag.2014.05.017

Sharma, M., Manoharlal, R., Puri, N., and Prasad, R. (2010). Antifungal curcumin induces reactive oxygen species and triggers an early apoptosis but prevents hyphae development by targeting the global repressor TUP1 in Candida albicans. Biosci. Rep. 30, 391-404. doi: 10.1042/BSR20090151

Sherry, L., Jose, A., Murray, C., Williams, C., Jones, B., Millington, O., et al. (2012). Carbohydrate derived fulvic acid: an in vitro investigation of a novel membrane active antiseptic agent against Candida albicans biofilms. Front. Microbiol. 3:116. doi: 10.3389/fmicb.2012.00116

Sherry, L., Rajendran, R., Lappin, D. F., Borghi, E., Perdoni, F., Falleni, M., et al. (2014). Biofilms formed by Candida albicans bloodstream isolates display phenotypic and transcriptional heterogeneity that are associated with resistance and pathogenicity. BMC Microbiol. 14:182. doi: 10.1186/1471-2180-14-182

Tyagi, P., Singh, M., Kumari, H., Kumari, A., and Mukhopadhyay, K. (2015). Bactericidal activity of curcumin I is associated with damaging of bacterial membrane. PLoS ONE 10:e0121313. doi: 10.1371/journal.pone.0121313

Vaux, D. L. (2012). Research methods: know when your numbers are significant. Nature 492, 180-181. doi: 10.1038/492180a

Wainwright, M., Maisch, T., Nonell, S., Plaetzer, K., Almeida, A., Tegos, G. P., et al. (2017). Photoantimicrobials-are we afraid of the light? Lancet Infect. Dis. 17, e49-e55. doi: 10.1016/S1473-3099(16)30268-7

Zhao, X., Oh, S. H., Cheng, G., Green, C. B., Nuessen, J. A., Yeater, K., et al. (2004). ALS3 and ALS8 represent a single locus that encodes a Candida albicans adhesin; functional comparisons between Als3p and Als1p. Microbiology 150, 2415-2428. doi: 10.1099/mic.0.26943-0

Conflict of Interest Statement: The authors declare that the research was conducted in the absence of any commercial or financial relationships that could be construed as a potential conflict of interest.

Copyright (c) 2017 Alalwan, Rajendran, Lappin, Combet, Shahzad, Robertson, Nile, Williams and Ramage. This is an open-access article distributed under the terms of the Creative Commons Attribution License (CC BY). The use, distribution or reproduction in other forums is permitted, provided the original author(s) or licensor are credited and that the original publication in this journal is cited, in accordance with accepted academic practice. No use, distribution or reproduction is permitted which does not comply with these terms. 